\title{
Tumor budding is associated with an increased risk of lymph node metastasis and poor prognosis in superficial esophageal adenocarcinoma
}

\author{
Michael S Landau ${ }^{1}$, Steven M Hastings ${ }^{1}$, Tyler J Foxwell ${ }^{1}$, James D Luketich ${ }^{2}$, \\ Katie S Nason ${ }^{2}$ and Jon M Davison ${ }^{1}$ \\ ${ }^{1}$ Department of Pathology, University of Pittsburgh School of Medicine, Pittsburgh, PA, USA and \\ ${ }^{2}$ Department of Cardiothoracic Surgery, University of Pittsburgh School of Medicine, Pittsburgh, PA, USA
}

\begin{abstract}
The treatment approach for superficial (stage T1) esophageal adenocarcinoma critically depends on the pre-operative assessment of metastatic risk. Part of that assessment involves evaluation of the primary tumor for pathologic characteristics known to predict nodal metastasis: depth of invasion (intramucosal vs submucosal), angiolymphatic invasion, tumor grade, and tumor size. Tumor budding is a histologic pattern that is associated with poor prognosis in early-stage colorectal adenocarcinoma and a predictor of nodal metastasis in T1 colorectal adenocarcinoma. In a retrospective study, we used a semi-quantitative histologic scoring system to categorize 210 surgically resected, superficial (stage T1) esophageal adenocarcinomas according to the extent of tumor budding (none, focal, and extensive) and also evaluated other known risk factors for nodal metastasis, including depth of invasion, angiolymphatic invasion, tumor grade, and tumor size. We assessed the risk of nodal metastasis associated with tumor budding in univariate analyses and controlled for other risk factors in a multivariate logistic regression model. In all, $41 \%$ (24 out of 59) of tumors with extensive tumor budding (tumor budding in $\geq 320 \mathrm{X}$ microscopic fields) were metastatic to regional lymph nodes, compared with $10 \%$ (12 out of 117 ) of tumors with no tumor budding, and $15 \%$ (5 out of 34 ) of tumors with focal tumor budding $(P<0.001)$. When controlling for all pathologic risk factors in a multivariate analysis, extensive tumor budding remains an independent risk factor for lymph node metastasis in superficial esophageal adenocarcinoma associated with a 2.5 -fold increase $(95 \% \mathrm{Cl}=1.1-6.3, P=0.039)$ in the risk of nodal metastasis. Extensive tumor budding is also a poor prognostic factor with respect to overall survival and time to recurrence in univariate and multivariate analyses. As an independent risk factor for nodal metastasis and poor prognosis after esophagectomy, tumor budding should be evaluated in superficial (T1) esophageal adenocarcinoma as a part of a comprehensive pathologic risk assessment.

Modern Pathology (2014) 27, 1578-1589; doi:10.1038/modpathol.2014.66; published online 25 April 2014
\end{abstract}

Keywords: adenocarcinoma; esophagus; tumor budding

In the majority of patients, surgically resected, superficial (T1) adenocarcinoma of the esophagus or gastroesophageal junction has a favorable survival outcome relative to more deeply invasive cancers. ${ }^{1}$ However, despite tumor that is confined to the mucosal or submucosal layers, up to $16 \%$ of

Correspondence: Dr JM Davison, MD, Department of Pathology, University of Pittsburgh School of Medicine, Presbyterian Hospital, Room A-610, 200 Lothrop Street, Pittsburgh, PA 15213, USA.

E-mail: davisonjm@upmc.edu

Received 27 November 2013; revised 25 February 2014; accepted 25 February 2014; published online 25 April 2014 patients with T1 esophageal adenocarcinoma will have nodal metastases identified at surgical resection. ${ }^{2-8}$ These patients have significantly worse prognosis. ${ }^{5,9}$

Based on a widespread consensus in the literature, ${ }^{2,3,5-8,10-16}$ submucosal invasion is routinely evaluated by staging endoscopic resection of superficial esophageal adenocarcinoma and is regarded as the paramount risk factor for nodal metastasis. ${ }^{17}$ However, there are other established risk factors for nodal metastasis, including angiolymphatic invasion,,$^{2,3,11,14,15,17-19}$ higher grade, ${ }^{2,3,8,11,15,17}$ and larger tumor size, ${ }^{3,11,14,17}$ which are also associated with nodal metastasis. 
In addition to these, tumor budding is another histologic feature that has been shown to be associated with lymph node metastasis or poor prognosis in other gastrointestinal neoplasms, including gastric, ${ }^{20}$ colorectal, ${ }^{21}$ and ampullary adenocarcinomas $^{22}$ and esophageal carcinomas. ${ }^{23,24} \mathrm{~A}$ tumor bud is defined as a detached cluster of fewer than five cells at the invasive front of a tumor. ${ }^{25}$ 'Tumor budding' is present when the number and density of buds exceed a threshold, with various scoring methods and thresholds proposed. At least some types of tumor budding are thought to be the morphologic manifestation of an epithelial-tomesenchymal transition during which tumor cells lose their intercellular attachments and acquire an invasive, mesenchymal phenotype that facilitates metastasis. ${ }^{21,26,27}$

Although tumor budding has been previously studied in esophageal carcinomas, little is known about its prognostic utility in superficial esophageal adenocarcinoma. An indication of its potential utility was suggested in a recent abstract in which tumor budding was found to be a risk factor for nodal metastasis and tumor recurrence in a cohort of 42 surgically resected superficial (stage T1) esophageal adenocarcinomas. ${ }^{28}$ Because there are multiple known pathologic predictors of nodal metastasis in superficial esophageal adenocarcinoma, it is important to evaluate tumor budding relative to these other prognostic factors to see whether it adds additional, independent prognostic information. Previous studies have not been sufficiently powered to do so. Therefore, the aims of this study are to document the prevalence and extent of tumor budding in surgically resected superficial esophageal adenocarcinoma and determine whether tumor budding is predictive of lymph node metastasis and survival when controlling for the effects of other important prognostic variables.

\section{Materials and methods}

\section{Case Selection}

We identified 210 patients with stage pT1 esophageal or gastroesophageal junction adenocarcinoma who underwent esophagectomy without induction therapy at the University of Pittsburgh Medical Center from 1996 to 2013 and had representative tumor slides available for review. Patients diagnosed with high-grade dysplasia only or staged as T2 or higher were not included, nor were patients with curative endoscopic resection of the tumor.

\section{Evaluation of Pathologic and Clinical Features}

The tumor slides from all 210 cases were reviewed and assessed for tumor size, tumor grade, submucosal invasion, angiolymphatic invasion, and tumor budding (MSL and JMD). An average of 3.9 blocks per tumor were evaluated (range 1-25), representing an average of 2.6 blocks per $\mathrm{cm}$ of tumor, not including deeper levels that were examined in some cases.

Tumor budding was semi-quantitatively scored for each tumor based on the maximum number of microscopic fields with tumor budding at the invasive front (illustrated in Figure 1). A tumor bud was defined as an isolated cluster of $<5$ tumor cells (including single-tumor cells) completely surrounded by stroma and lacking gland lumen formation. A 'tumor budding field' was defined as a 20X microscopic field (Olympus BX45, Olympus Plan N 20X objective lens; measuring $0.785 \mathrm{~mm}^{2}$ ) with five or more tumor buds based on the definition of Ueno et al. ${ }^{25}$ We counted the number of budding fields in each tumor section and classified individual cases using the following cutoffs established $a$ priori: no budding fields, 1 to 2 budding fields, or 3 or more budding fields based on the scoring methodology of Ohike et al. ${ }^{22}$ To compensate for differences in sampling density (number of tumor blocks per $\mathrm{cm}$ of tumor), the final budding score for each case was based on the tumor section with the greatest number of tumor budding fields.

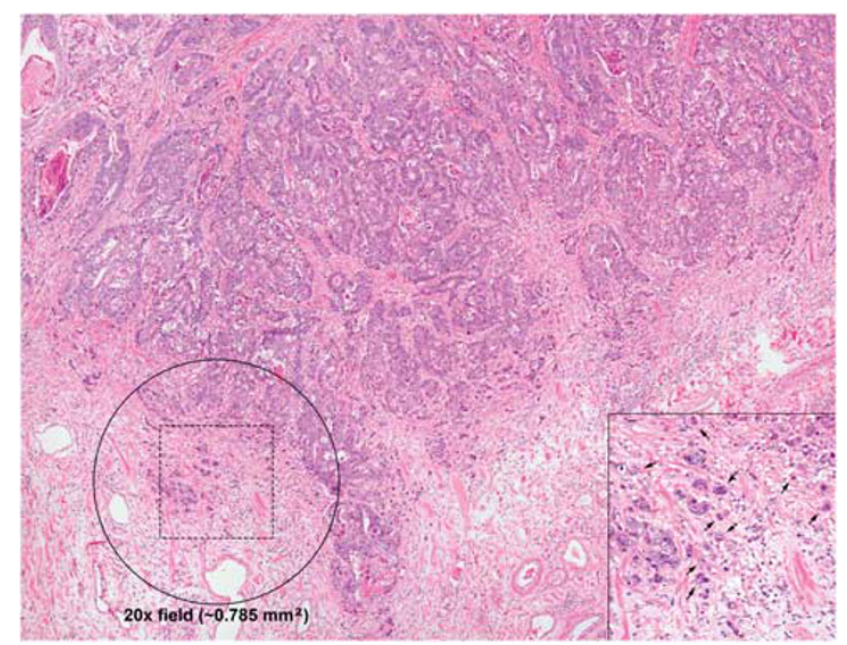

Figure 1 Scoring of tumor budding. This figure illustrates the semi-quantitative scoring criteria used to assess tumor budding in each case. Even at relatively low magnification, areas that are likely to represent tumor budding are apparent due to the indistinct border between tumor and stroma at the advancing edge of the tumor. The circle represents the approximate size of a single 20X high-power field $\left(0.785 \mathrm{~mm}^{2}\right)$. For this study, we counted the number of 20X fields with more than five tumor buds ('tumor budding fields'). Individual tumor buds were defined as clusters of fewer than five tumor cells lacking gland lumen formation at the advancing edge of the tumor. Several of the individual tumor buds in the hashed square are indicated by arrows (inset). As detailed in the Materials and methods, cases were classified as no tumor budding (no 20X fields with more than five tumor buds); focal tumor budding (1-2 20X budding fields); or extensive tumor budding ( $\geq 320 \mathrm{X}$ budding fields) according to Ohike et al. ${ }^{22}$ 
Tumor size was determined based on the measurement reported in the original surgical pathology report and then confirmed by microscopic examination and review of gross photographs when necessary. For small tumors or tumors not grossly apparent, the size was determined by measuring the largest microscopic cross-sectional size. For multifocal tumors, the size of the largest single focus was recorded. We used the median tumor size in our cohort $(2.0 \mathrm{~cm})$ as a cutoff for assessing risk of nodal metastasis. This cutoff has been previously reported as a risk factor for nodal metastasis. ${ }^{3}$

Tumor grade was determined based on the current College of American Pathologists reporting guidelines for esophageal adenocarcinoma and the 2010 World Health Organization criteria for grading colorectal adenocarcinoma: ${ }^{29}$ tumors composed of $>95 \%$ isolated tubular glands were considered well differentiated, those composed of 50-95\% glandular structures were considered moderately differentiated, and those composed of $<50 \%$ glands (ie, $>50 \%$ solid growth or individual cells) were considered poorly differentiated.

Angiolymphatic invasion was defined as tumor cells within endothelium-lined vascular or lymphatic spaces on H\&E section within or adjacent to the primary tumor. Angiolymphatic invasion was not scored in the periesophageal or perigastric fat. Borderline cases were considered negative.

Submucosal invasion (stage T1b) was defined as definite extension of the tumor beyond the true muscularis mucosae and into the submucosa; tumors not satisfying this criterion were classified as intramucosal (pT1a). We further stratified depth of invasion according to Liu et $a:^{4}$ intramucosal adenocarcinomas were classified as T1a-superficial, if they were confined to the lamina propria, otherwise they were classified as T1a-deep. Submucosal adenocarcinomas were classified as T1b-superficial, if they invaded less than half the thickness of the submucosa at the point of invasion, and T1bdeep, if they invaded more than half the submucosal thickness.

The tumor location, presence of lymph node metastases, and the total lymph node count were determined based on the original surgical pathology report and confirmed on review. Tumors involving the esophagus or proximal stomach that invaded across the anatomic gastroesophageal junction were categorized as gastroesophageal junction adenocarcinomas. Tumors located in the esophagus that did not involve the anatomic gastroesophageal junction were categorized as esophageal. We did not include primary gastric carcinomas in the study. Barrett's esophagus was considered present if esophageal intestinal metaplasia was confirmed histologically in the esophagectomy specimen or in pre-operative biopsies. Age and sex, type of operation, and presence of Barrett's esophagus were recorded from surgical pathology reports and the patient's electronic medical record. This study was approved by the University of Pittsburgh Institutional Review Board.

\section{Statistical Analysis}

Differences in categorical variables were analyzed by $\chi^{2}$ or Fischer's exact tests. Differences in continuous variables were evaluated by MannWhitney $U$-test. A multivariate logistic regression model was used to identify independent predictors of nodal metastasis. Variables that were found to be statistically significant in the univariate analysis were included in the multivariate analysis. Overall survival was determined from the time of esophagectomy until death-as documented in the clinical record or Social Security Death Index-or the time of last known clinical follow-up. Time to recurrence was defined as the time from esophagectomy to first recurrence or the last clinical evaluation for recurrence. Local, regional, and distant recurrences were all included. The survival estimates for tumor budding groups were characterized using KaplanMeier curves, with statistical differences tested using the log-rank test. Cox proportional hazards analysis was used to control for other known predictors of survival in multivariate analysis. Patients who died as a result of complications from surgery (survival $<3$ months) were excluded from the analysis. All tests were two-sided and statistical significance was defined as a $P$-value $<0.05$.

\section{Results}

\section{Patient Characteristics}

The median age of patients included in the study was 66 years (interquartile range $60-74$ years) and the majority $(n=179 ; 85 \%)$ were men. Most patients $(n=176 ; 85 \%)$ underwent minimally invasive esophagectomy ${ }^{30}$ and the remainder underwent an open $(n=22 ; 11 \%)$ or hybrid procedure $(n=8 ; 4 \%)$, most often a transhiatal esophagectomy $(n=15$; $7 \%$ ); the operative approach was not specified in the available medical record for 4 patients. Margins of resection were negative in all cases. There were $125(60 \%)$ tumors located at the gastroesophageal junction and $85(40 \%)$ tumors located in the esophagus (79 lower esophagus, 6 middle esophagus). Barrett's esophagus was present in $92 \%(n=193)$ of patients.

\section{Overall Pathologic Characteristics}

Two-thirds of the superficial esophageal adenocarcinomas $(n=138,66 \%)$ invaded into the submucosa (T1b), almost equally divided between T1b-superficial and T1b-deep (Table 1). The remaining intramucosal adenocarcinomas (T1a) were almost equally divided between T1a-superficial and T1a-deep. Our assessment of $\mathrm{T}$ stage (T1a vs T1b) 
Table 1 Lymph node status associated with pathologic features

\begin{tabular}{|c|c|c|c|c|}
\hline & All cases, $\mathrm{N}$ & Node negative, N (\%) & Node positive, N (\%) & $\mathrm{P}$-value \\
\hline \multicolumn{5}{|l|}{ Tumor stage } \\
\hline T1a (intramucosal) & 72 & $69(96)$ & $3(4)$ & \multirow[t]{2}{*}{$<0.001$} \\
\hline T1b (submucosal) & 138 & $100(72)$ & $38(28)$ & \\
\hline \multicolumn{5}{|l|}{ Depth of invasion, intramucosal only } \\
\hline T1a-superficial & 35 & $34(97)$ & $1(3)$ & \multirow[t]{2}{*}{1.000} \\
\hline T1a-deep & 37 & 35 (95) & $2(5)$ & \\
\hline \multicolumn{5}{|l|}{ Depth of invasion, submucosal only } \\
\hline T1b-superficial & 72 & $57(79)$ & $15(21)$ & \multirow[t]{2}{*}{0.049} \\
\hline T1b-deep & 66 & $43(65)$ & $23(35)$ & \\
\hline \multicolumn{5}{|l|}{ Lymph node stage } \\
\hline No (0 positive lymph nodes) & 169 & $169(100)$ & $0(0)$ & \multirow{4}{*}{ NA } \\
\hline N1 (1-2 positive lymph nodes) & 26 & $0(0)$ & $26(100)$ & \\
\hline N2 (3-6 positive lymph nodes) & 10 & $0(0)$ & $10(100)$ & \\
\hline N3 (>6 positive lymph nodes) & 5 & $0(0)$ & $5(100)$ & \\
\hline Lymph nodes examined (median, interquartile range) & $20(13-28)$ & $19(14-28)$ & $20(13-28)$ & 0.969 \\
\hline \multicolumn{5}{|l|}{ WHO tumor grade } \\
\hline Well differentiated & 40 & $38(95)$ & $2(5)$ & \multirow{3}{*}{0.003} \\
\hline Moderately differentiated & 138 & $111(80)$ & $27(20)$ & \\
\hline Poorly differentiated & 32 & $20(62)$ & $12(38)$ & \\
\hline \multicolumn{5}{|l|}{ Tumor budding } \\
\hline None & 117 & $105(90)$ & $12(10)$ & \multirow{3}{*}{$<0.001$} \\
\hline Focal (1-2 budding fields) & 34 & $29(85)$ & $5(15)$ & \\
\hline Extensive ( $\geq 3$ budding fields) & 59 & $35(59)$ & $24(41)$ & \\
\hline \multicolumn{5}{|l|}{ Tumor budding } \\
\hline None or focal ( $<3$ budding fields) & 151 & $134(89)$ & $17(11)$ & \multirow[t]{2}{*}{$<0.001$} \\
\hline Extensive ( $\geq 3$ budding fields) & 59 & $35(59)$ & $26(41)$ & \\
\hline \multicolumn{5}{|l|}{ Angiolymphatic invasion } \\
\hline Absent & 174 & $150(86)$ & $24(14)$ & \multirow[b]{2}{*}{$<0.001$} \\
\hline Present & 36 & $19(53)$ & $17(47)$ & \\
\hline \multicolumn{5}{|l|}{ Tumor size $(\mathrm{cm})$} \\
\hline$<2$ & 105 & $97(92)$ & $8(8)$ & \multirow[t]{2}{*}{$<0.001$} \\
\hline$\geq 2$ & 105 & $72(69)$ & $33(31)$ & \\
\hline
\end{tabular}

agreed with the original diagnosis in 199 out of $210(95 \%)$ of cases. On review, six cases were upstaged (T1a to T1b) and five cases were downstaged (T1b to T1a).

Nearly, two-thirds (138 out of 210) of cases were graded as moderately differentiated and 15\% (32 out of 210) were graded poorly differentiated (Table 1). Angiolymphatic invasion was identified in 36 out of 210 cases (17\%; Table 1 ). The median tumor size in our cohort was $1.95 \mathrm{~cm}$ (interquartile range $=1.0-$ $3.0 \mathrm{~cm})$.

Lymph node metastases were present in $20 \%$ $(n=41)$ of all cases. Of those with node metastases, the majority of cases (26 out of $41,63 \%$ ) had 1-2 positive lymph nodes (stage pN1). The median number of lymph nodes examined at esophagectomy was 20 (interquartile range $=13-28$ ) and did not significantly differ between the node-positive and the node-negative groups (Table 1).

Some degree of tumor budding was seen in 93 out of 210 cases. It was focal (1-2 20X fields) in 16\% $(n=34)$ and extensive $(\geq 3$ 20X fields) in $28 \%$ $(n=59)$.

\section{Association between Other Tumor Characteristics and Tumor Budding}

Ninety-five percent of superficial esophageal adenocarcinoma with extensive tumor budding ( $\geq 3$ 20X budding fields) invaded either the superficial or deep submucosa compared with only $54 \%$ of those with none or focal budding $(P<0.001$, Table 2). Likewise, tumors with extensive tumor budding were more likely to be poorly differentiated, angioinvasive, and larger than $2 \mathrm{~cm}(P<0.001$ for all, Table 2). The association with tumor grade is noteworthy. Although extensive tumor budding was more common in high-grade tumors (present in 23 out of 32), it was also present in 36 out of 178 wellto-moderately differentiated tumors graded according to the College of American Pathologists/World 
Table 2 Pathologic features according to tumor budding status

\begin{tabular}{|c|c|c|c|}
\hline & \multicolumn{2}{|c|}{ Tumor budding } & \multirow[b]{2}{*}{$\mathrm{P}$-value } \\
\hline & $\begin{array}{l}\text { None or focal } \\
\text { (0-2 budding } \\
\text { fields) } \\
\mathrm{N}(\%)\end{array}$ & $\begin{array}{l}\text { Extensive } \\
\text { ( } \geq 3 \text { budding } \\
\text { fields) } \\
\mathrm{N}(\%)\end{array}$ & \\
\hline \multicolumn{4}{|l|}{ Depth of invasion (T stage) } \\
\hline $\begin{array}{l}\text { Intramucosal (T1a), } \\
\text { superficial }\end{array}$ & $34(23)$ & $1(2)$ & \\
\hline Intramucosal (T1a), deep & $35(23)$ & $2(3)$ & $<0.001$ \\
\hline $\begin{array}{l}\text { Submucosal (T1b), } \\
\text { superficial }\end{array}$ & $56(37)$ & $16(27)$ & \\
\hline Submucosal (T1b), deep & $26(17)$ & $40(68)$ & \\
\hline \multicolumn{4}{|l|}{ WHO tumor grade } \\
\hline Well differentiated & $39(26)$ & $1(2)$ & \\
\hline Moderately differentiated & $103(68)$ & $35(59)$ & \\
\hline Poorly differentiated & $9(6)$ & $23(39)$ & $<0.001$ \\
\hline \multicolumn{4}{|l|}{ Angiolymphatic invasion } \\
\hline Absent & $137(91)$ & $37(63)$ & \\
\hline Present & $14(9)$ & $22(37)$ & $<0.001$ \\
\hline \multicolumn{4}{|l|}{ Tumor size $(\mathrm{cm})$} \\
\hline$<2$ & $90(60)$ & $15(25)$ & \\
\hline$\geq 2$ & $61(40)$ & $44(75)$ & $<0.001$ \\
\hline
\end{tabular}

Health Organization criteria (Table 2; examples in Figure 2).

\section{Extensive Tumor Budding and Angiolymphatic Invasion are Independent Risk Factors for Lymph Node Metastasis}

We next evaluated the association of primary tumor characteristics with the prevalence of nodal metastasis. Submucosal invasion $(P<0.001)$, higher tumor grade $(P=0.003)$, extensive tumor budding $(P<0.001)$, angiolymphatic invasion $(P<0.001)$, and tumor size $\geq 2 \mathrm{~cm}(P<0.001)$ all were associated with a significantly higher prevalence of nodal metastasis (Table 1). For instance, 41\% (24 out of 59) of tumors with extensive tumor budding were metastatic to regional lymph nodes, compared with $10 \%$ (12 out of 117) of tumors with no tumor budding and $15 \%$ (5 out of 34 ) of tumors with focal tumor budding (Table 1). The difference in nodal metastasis between tumors with none vs focal tumor budding (one to two fields) was not significant $(P=0.538)$. We therefore used extensive tumor budding (at least three tumor budding fields) as a cutoff in the subsequent multivariate analysis as this was associated with a significantly higher frequency of nodal metastasis compared with all tumors without extensive budding $(P<0.001$, Table 1$)$.

We also further evaluated depth of invasion and found that there was no difference in the prevalence of nodal metastasis when comparing superficial intramucosal (T1a) esophageal adenocarcinoma to deep T1a esophageal adenocarcinoma (Table 1).
Given the limited number of cases with metastases in this group $(n=3)$, this finding was not unexpected. However, depth of invasion into the submucosa did show a weak association with the prevalence of node metastasis $(P=0.049)$ as $37 \%$ of esophageal adenocarcinoma with deep submucosal invasion had node metastasis compared with $22 \%$ of superficial submucosal esophageal adenocarcinoma (Table 1). Given these findings we categorized depth of invasion for the subsequent multivariate analysis as T1a, T1b-superficial, and T1b-deep.

Because of the significant association between tumor budding and other variables known to predict lymph node metastasis, we performed a multivariate logistic regression analysis to determine which risk factors were independent predictors of nodal metastasis. In the univariate logistic regression, the presence of extensive tumor budding increased the odds of nodal metastasis by 5.4 -fold (95\% confidence interval $=2.6-11.1, P<0.001$, Table 3). Similarly, the odds of nodal metastasis were significantly increased in the presence of submucosal invasion (either superficial or deep), high tumor grade, angiolymphatic invasion, and tumor size $\geq 2 \mathrm{~cm}$ (Table 3 ). In the multivariate logistic regression, extensive tumor budding ( $\geq 3$ budding fields) was independent of other risk factors and associated with a 2.5 -fold (95\% confidence interval $=1.1-6.3$, $P=0.039)$ increased risk of nodal metastasis (Table 3). Angiolymphatic invasion $(P=0.049)$ also remained an independent predictor when controlling for other variables. Tumor size $\geq 2 \mathrm{~cm}$ showed a trend toward increased risk of nodal metastasis $(P=0.061)$, but depth of invasion $(P=0.298)$ and high tumor grade $(P=0.716)$ did not.

\section{The Effect of Tumor Budding on the Risk of Lymph Node Metastasis in Combination with Other Risk Factors}

To illustrate how tumor budding modifies the risk of nodal metastasis associated with other predictors, we classified tumors using combinations of risk factors (Figure 3). When tumors are grouped based on tumor grade and tumor budding, 15 out of 36 $(42 \%)$ of low-grade (well-to-moderately differentiated) tumors with extensive tumor budding were node positive compared with only 14 out of 142 $(10 \%)$ of low-grade tumors without extensive budding $(P<0.001$, Figure 3). Interestingly, we found no difference in the rate of node metastasis between low-grade tumors with extensive tumor budding and high-grade tumors $(P=0.726)$.

Although superficial submucosal invasion (T1bsuperficial) was associated with a $21 \%$ rate of nodal metastasis overall (Table 1 ), the rate of nodal metastasis is significantly influenced by tumor budding. Eight of sixteen (50\%) T1b-superficial cancers with extensive tumor budding were metastatic vs only 7 out of $56(13 \%)$ of those without 

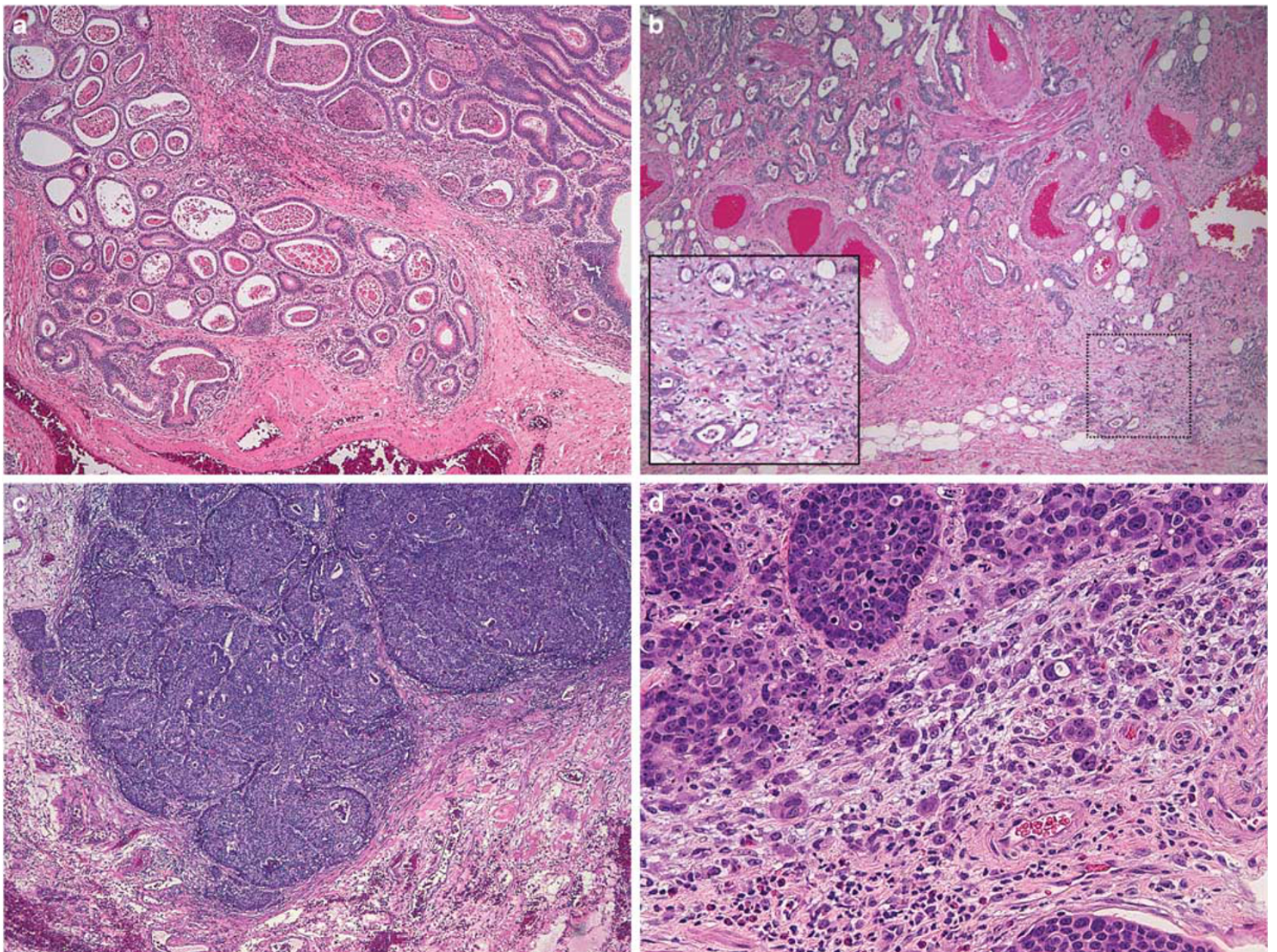

Figure 2 Representative examples of tumor grade and budding. (a) Low-grade tumor without tumor budding. The well-formed glands with luminal necrosis invade the submucosa and are surrounded by a mild inflammatory infiltrate at low power (approximately $40 \mathrm{X}$ magnification). (b) A predominantly low-grade adenocarcinoma with tumor budding. Budding is best seen in the inset at the advanced edge of the tumor as it infiltrates the submucosal fat (40X magnification, inset 200X magnification). (c) A poorly differentiated adenocarcinoma that lacks tumor budding. There is a fairly distinct 'pushing' interface between the advancing edge of the tumor and the surrounding stroma (40X magnification). (d) A poorly differentiated adenocarcinoma shows prominent tumor budding in this highpower field (200X magnification).

extensive budding $(P=0.003$, Figure 3$)$. Intramucosal cancers seldom metastasized; consequently, there was insufficient statistical power for comparison of subgroups.

In cases without angiolymphatic invasion, 13 out of 37 (35\%) with tumor budding were node positive, compared with only 11 out of 137 (8\%) with neither risk factor $(P<0.001$, Figure 3). Last, extensive tumor budding was associated with a significantly increased rate of nodal metastasis in small T1 esophageal adenocarcinoma $(\leq 2 \mathrm{~cm})$ and large T1esophageal adenocarcinoma $(>2 \mathrm{~cm}$, Figure 3$)$.

\section{Tumor Budding is a Poor Prognostic Factor with Respect to Overall Survival and Time to Recurrence}

Out of the entire cohort of 210 patients, 194 were included in the survival analysis. There were 16 patients excluded because they died within 3 months of surgery $(N=13)$ or had fewer than 3 months clinical follow-up recorded $(N=3)$. Among the included patients, there were 72 deaths. The median follow-up interval was 44 months.

Because of the association with nodal status, tumor budding is expected to be a poor prognostic factor. When patients were stratified based on the extent of tumor budding, there was a significant difference in survival associated with the extent of tumor budding. Seventy-nine percent of patients with no tumor budding were alive at 5 years, compared with seventy-one percent of patients with focal budding and thirty-seven percent of those with extensive tumor budding $(P<0.0001$, Figure 4a). In univariate Cox proportional hazards analysis, extensive tumor budding, submucosal invasion (T stage), higher $\mathrm{N}$ stage, higher patient age, and undergoing an operation other than minimally 
Table 3 Univariate and multivariate analysis of histologic predictors of node metastasis

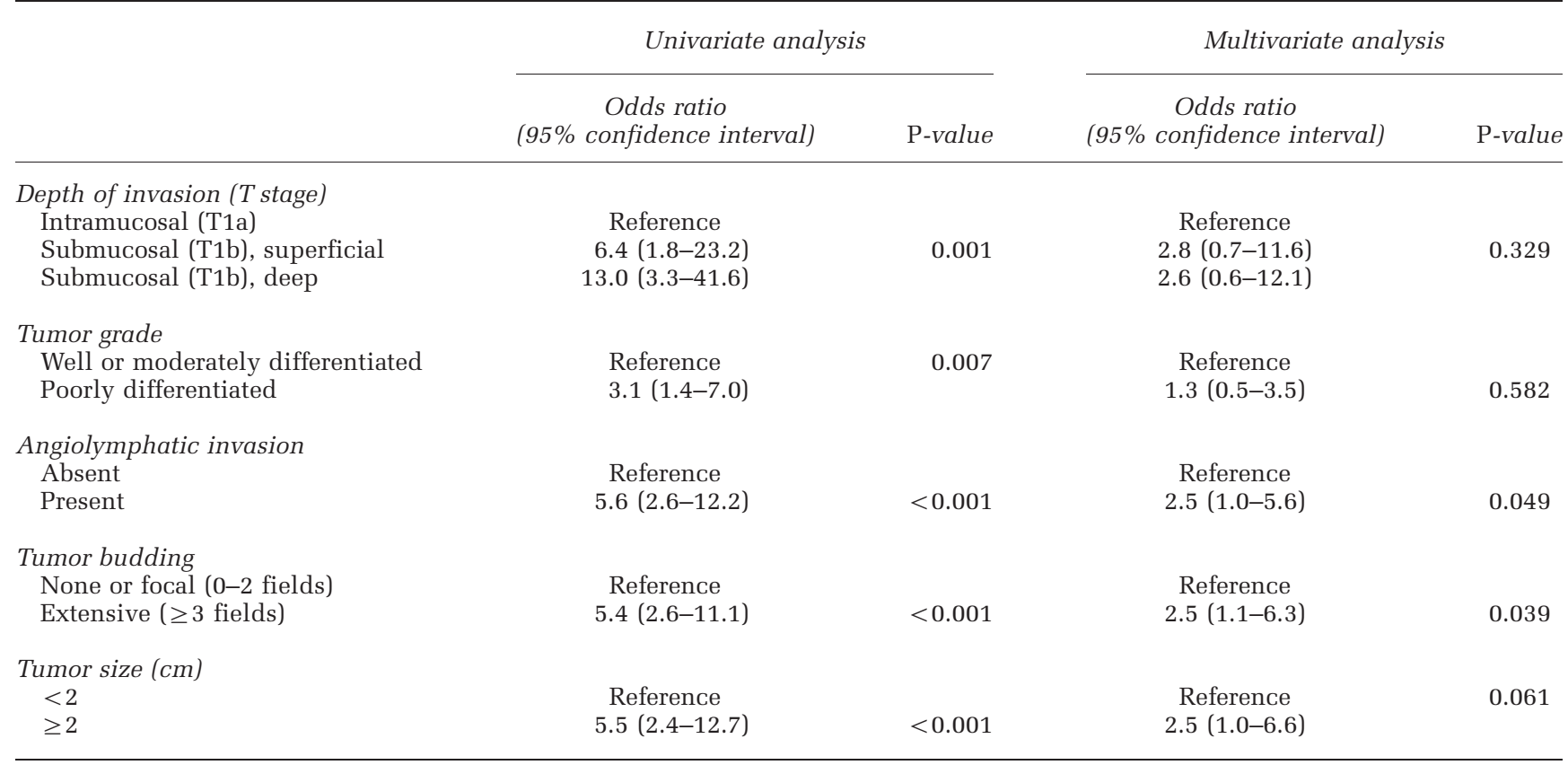

invasive esophagectomy were all significant adverse prognostic factors (data not shown). We performed a multivariate analysis to determine whether the negative effect of tumor budding on survival was independent of these other factors. Extensive tumor budding was associated with a 3.3-fold increased risk of death $(95 \%$ confidence interval $=1.5-7.4$, $P=0.004$ ), after controlling for these other prognostic variables in the multivariate analysis.

Time to recurrence is a more specific indicator of aggressive tumor behavior in superficial gastroesophageal cancer because of its relatively favorable prognosis. In the 194 patients, there were 31 recurrences (25 with distant metastatic recurrence, 2 with only regional lymph node recurrence and 4 with only local anastomotic recurrences). Median time to last clinical evaluation for recurrence was 30 months. In patients with a documented recurrence, the median time to recurrence was 14 months (range 4-50 months). At 24 months, only $5 \%$ of patients without tumor budding had recurrence. In comparison, $19 \%$ of those with focal budding and $36 \%$ of those with extensive tumor budding had developed recurrence by 24 months $(P<0.0001$, Figure $4 \mathrm{~b})$. When patients with focal budding were compared with those with no tumor budding, the modest difference in time to recurrence was not significant $(P=0.096)$. In univariate analyses, both submucosal invasion ( $\mathrm{T}$ stage) and higher $\mathrm{N}$ stage were risk factors for tumor recurrence (data not shown), so we also performed a multivariate Cox proportional hazards analysis to control for these factors. Extensive tumor budding was associated with a 3.2 -fold increased risk of recurrence (95\% confidence interval $=1.4-7.0, P=0.005$ ), independent of $\mathrm{T}$ and $\mathrm{N}$ stage in the multivariate analysis.

\section{Discussion}

Predicting nodal metastasis is of critical importance in superficial esophageal adenocarcinoma because this risk assessment is the primary determinant of the therapeutic approach. Therapy can range from endoscopic resection of the tumor with close followup for low-risk tumors to neoadjuvant chemotherapy and radiation followed by esophagectomy for those thought to possess a high metastatic risk. The results of our study show that tumor budding is an independent predictor of nodal metastasis in superficial esophageal adenocarcinoma. In addition, it is a poor prognostic factor for overall survival and time to recurrence independent of other important survival factors.

Previously, tumor budding has been extensively studied in colorectal adenocarcinoma, where it is an important prognostic feature associated with lymph node metastasis, local recurrence, and cancerrelated death particularly in American Joint Committee on Cancer stage $1^{25,31-39}$ and stage $2^{21,37,40-43}$ disease. In a widely cited study, tumor budding along with high tumor grade and vascular invasion were independent prognostic features associated with lymph node metastasis in submucosally invasive colorectal adenocarcinoma. ${ }^{25}$ Tumor budding has also been shown to be an independent prognostic factor associated with overall survival in esophageal squamous carcinomas. ${ }^{23,44}$ 
a

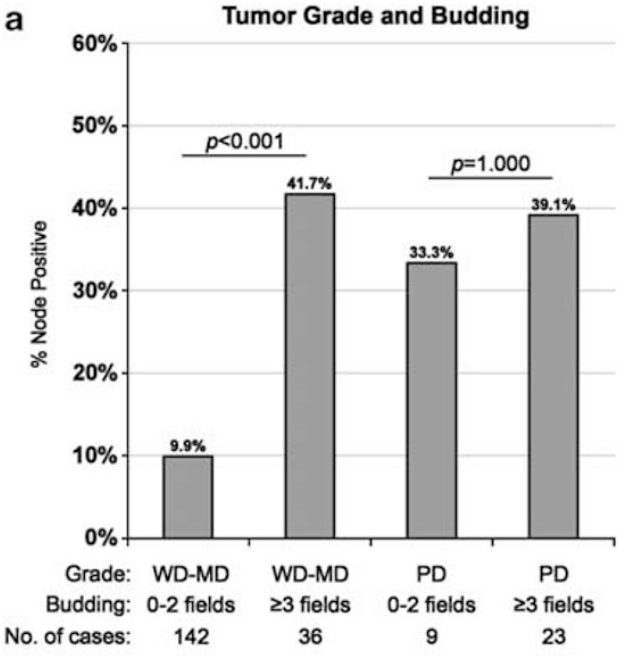

b

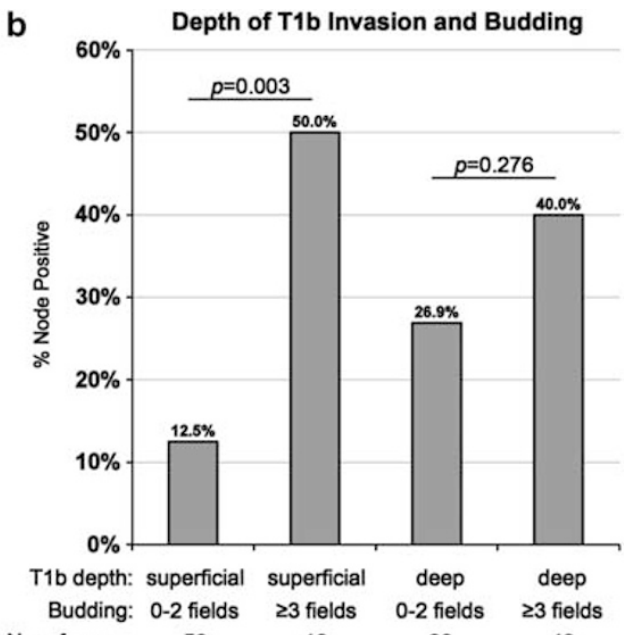

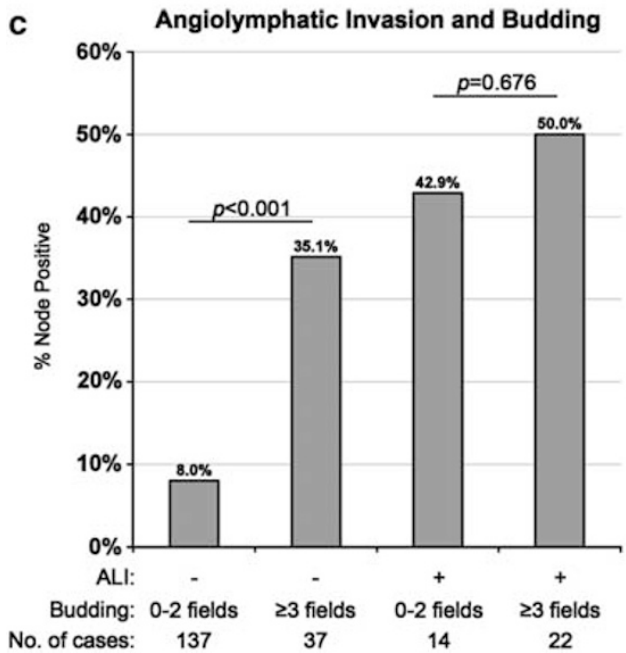$$
\begin{array}{lllll}
\text { No. of cases: } & 137 & 37 & 14 & 22
\end{array}
$$

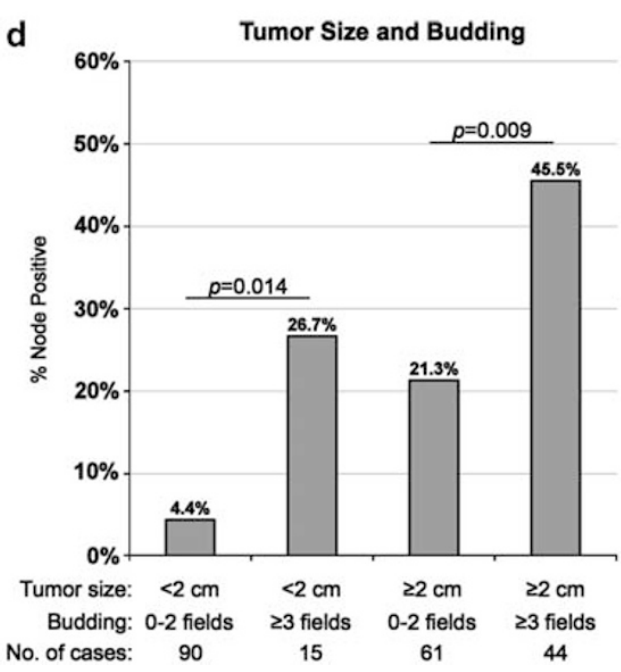

Figure 3 Bar graphs illustrating the prevalence of node metastasis in cases classified based on the presence of tumor budding and tumor grade (a), depth of submucosal invasion (b), angiolymphatic invasion (c) and tumor size (d).

a

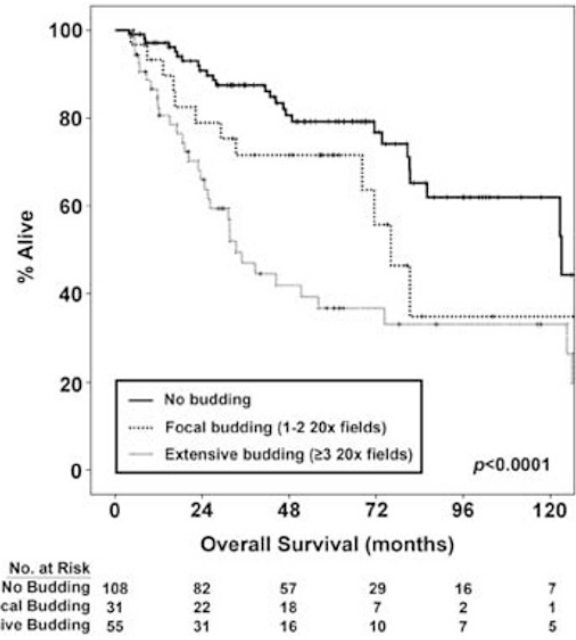

b

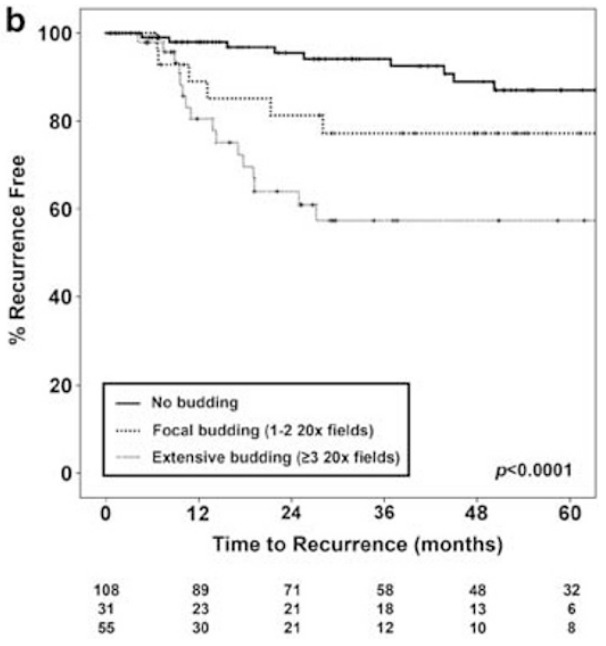

Figure 4 Kaplan-Meier curves for overall survival and time to recurrence in patients stratified by the extent of tumor budding. The graphs demonstrate significant differences in the survival functions. Extensive tumor budding was associated with worse overall survival (a) and accelerated time to recurrence (b). 
With regard to tumor budding in esophageal adenocarcinoma, one prior study found tumor budding to be a prognostic factor for overall survival in a cohort of patients with esophageal cancer, which included 287 adenocarcinomas and 69 squamous cell carcinomas. ${ }^{24}$ This association with outcome persisted even when controlling for other prognostic factors in a multivariate analysis. However, that study did not evaluate the association of tumor budding with nodal metastasis and included only 38 T1 tumors. A more recent study published in abstract form showed that tumor budding was associated with increased likelihood of tumor recurrence and nodal metastasis in $42 \mathrm{~T} 1$ esophageal adenocarcinomas. ${ }^{28}$ Although that study assessed superficial esophageal adenocarcinomas for other histologic risk factors (tumor size, grade, angioinvasion, and depth of invasion), it was not sufficiently powered to evaluate whether tumor budding was an independent prognostic factor when controlling for other predictors of nodal metastasis and recurrence.

One challenge in studying the prognostic significance of tumor budding is the absence of a universally accepted definition that is both qualitative (small clusters of cells at the invasive front) and quantitative (how much budding is needed to increase the risk). The most common method proposed by Ueno uses a cutoff of five or more individual buds in a 20X field (area of $0.785 \mathrm{~mm}^{2}$ ) at the invasive front. ${ }^{25}$ Other studies have proposed more labor-intensive quantitative scoring systems, requiring precise numerical scoring of multiple high-power fields followed by averaging of tumor bud counts. ${ }^{32,45-48}$ Although this is likely to produce an accurate description of the extent and variability of tumor budding, we feel that a simpler approach is required for routine clinical practice.

We therefore selected a semi-quantitative method previously used to evaluate ampullary adenocarcinomas that relies on counting the number of $20 \mathrm{X}$ fields with more than five tumor buds. ${ }^{22}$ In that study, tumors with three or more 20X tumor budding fields were more likely to metastasize than those with fewer. The general utility of this scoring method and quantitative cutoff is supported by our finding in superficial esophageal adenocarcinoma that tumors with focal tumor budding (one to two budding fields) did not have a greater risk of lymph node metastasis than those with no budding, but tumors with three or more budding fields had a significantly higher risk of nodal metastasis and worse survival outcomes. It is worth noting that focal tumor budding did show a trend toward a higher risk of recurrence, but the difference in survival compared with those with no tumor budding was not significant. It may be prudent to regard focal tumor budding as an indeterminate risk factor in T1 esophageal adenocarcinoma.

The issue of whether to score tumor budding on the basis of H\&E-stained sections or a broad spectrum keratin immunostain has been discussed. ${ }^{45}$ In preparation for this study, we did review serial H\&E sections along with sections stained with pancytokeratin to confirm our impression of tumor budding (data not shown). This anecdotal experience convinced us that in the large majority of cases, a keratin stain would not be required to identify and score tumor budding and it is unlikely in clinical practice that pathologists would prefer to obtain keratin immunostains in all cases as a condition for scoring tumor budding. We, therefore, elected not to use a keratin stain in this study. We would echo the practical suggestion made by Mitrovic et al. that a pankeratin immunostain be reserved for difficult cases (ie, in the presence of abundant inflammation) to confirm the histologic identification and quantitation of tumor budding. ${ }^{49}$

The College of American Pathologists/World Health Organization criteria for grading adenocarcinomas of the gastrointestinal tract are based on glandular architecture. Using these criteria, extensive tumor budding may increase the tumor grade (ie, if the tumor budding pattern represented over half of the tumor it would be graded as poorly differentiated). However, only $38 \%$ of the cases in our series with extensive tumor budding were poorly differentiated by the College of American Pathologists/World Health Organization criteria. The remainder were low grade (well or moderately differentiated), illustrating that a tumor can show prominent glandular architecture and have extensive tumor budding by the Ohike criteria. ${ }^{22}$ In fact, in our subgroup analysis, we show that low-grade tumors with tumor budding have a similar risk of nodal metastasis as poorly differentiated adenocarcinomas. Because tumor budding and architectural grade are interrelated, future studies should evaluate the utility of a grading system that combines glandular architecture and tumor budding.

It may be surprising to some that depth of invasion was not an independent risk factor for nodal metastasis because it is generally regarded as an essential predictor of nodal metastasis in routine clinical practice. Our data confirm numerous studies that show submucosal invasion is a risk factor for nodal metastasis ${ }^{4,8,11,16,19,50-53}$ when analyzed as a single variable. Because of the number of cases in our series, we were able to control for the most common additional risk factors and show that the risk associated with submucosal invasion is significantly influenced by other variables. In other words, tumors that invade into the submucosa are more likely to be larger than $2 \mathrm{~cm}$, to be poorly differentiated, and to show angiolymphatic invasion..$^{3,4}$ We also show that they are also more likely to have extensive tumor budding. These observations beckon for a system of risk stratification that accounts for multiple variables in addition to depth of invasion. Such an approach could be clinically useful, as suggested by studies showing it is possible to safely treat carefully selected submucosal esophageal 
adenocarcinoma based on the absence of other highrisk pathologic features. ${ }^{54}$

We noted that extensive tumor budding is almost never the only risk factor for nodal metastasis in a given tumor, yet our multivariate analysis demonstrates that its predictive significance is independent of other commonly accepted risk factors. We illustrate how the relationship between other risk factors and nodal metastasis is modified by the extent of tumor budding such that T1b-superficial, low-grade tumors, and tumors without angiolymphatic invasion had dramatically higher rates of nodal metastasis in the presence of extensive tumor budding.

We chose to focus on the subset of patients with T1 esophageal adenocarcinoma in this study because predicting nodal metastasis in this patient population is of utmost clinical relevance. The significance of tumor budding may be different in more advanced esophageal adenocarcinoma with a significantly higher likelihood of nodal metastasis. Tumor budding and other pathologic risk factors for nodal metastasis could be assessed before definitive therapy in endoscopic resection specimens or in biopsies. Intratumoral budding identified in biopsy specimens of rectal carcinoma was recently shown to associate with poor response to neoadjuvant treatment. ${ }^{55}$ Our study did not address this question and future studies to evaluate the predictive ability of these pathologic features in the pre-operative setting are warranted.

Last, in addition to tumor budding, we focused this report on the most commonly cited pathologic predictors of nodal metastasis in superficial esophageal adenocarcinoma. We did not evaluate all previously reported prognostic factors such as human epidermal growth factor receptor 2 amplification, epidermal growth factor receptor amplification, inflammatory host response, and perineural invasion, among others. To incorporate additional variables into a multivariate analysis would require a larger number of cases.

In summary, we have shown that tumor budding is an independent risk factor for nodal metastasis in superficial esophageal adenocarcinoma when evaluated by a semi-quantitative scoring method on routine histologic sections. Tumor budding should be evaluated in superficial esophageal adenocarcinoma as part of a comprehensive prognostic assessment.

\section{Acknowledgments}

The project described was supported in part by Award Number K07CA151613 (KSN) from the National Cancer Institute.

\section{Disclosure/conflict of interest}

The authors declare no conflict of interest.

\section{References}

1 Rice TW, Rusch VW, Ishwaran $\mathrm{H}$, et al. Worldwide Esophageal Cancer C. Cancer of the esophagus and esophagogastric junction: data-driven staging for the seventh edition of the American Joint Committee on Cancer/International Union Against Cancer Cancer Staging Manuals. Cancer 2010;116:3763-3773.

2 Barbour AP, Jones M, Brown I, et al. Risk stratification for early esophageal adenocarcinoma: analysis of lymphatic spread and prognostic factors. Ann Surg Oncol 2010;17:2494-2502.

3 Leers JM, DeMeester SR, Oezcelik A, et al. The prevalence of lymph node metastases in patients with T1 esophageal adenocarcinoma a retrospective review of esophagectomy specimens. Ann Surg 2011;253: 271-278.

4 Liu L, Hofstetter WL, Rashid A, et al. Significance of the depth of tumor invasion and lymph node metastasis in superficially invasive (T1) esophageal adenocarcinoma. Am J Surg Pathol 2005;29:1079-1085.

5 Rice TW, Blackstone EH, Goldblum JR, et al. Superficial adenocarcinoma of the esophagus. J Thorac Cardiovasc Surg 2001;122:1077-1090.

6 Stein HJ, Feith M, Bruecher BL, et al. Early esophageal cancer: pattern of lymphatic spread and prognostic factors for long-term survival after surgical resection. Ann Surg 2005;242:566-573.

7 van Sandick JW, van Lanschot JJ, ten Kate FJ, et al. Pathology of early invasive adenocarcinoma of the esophagus or esophagogastric junction: implications for therapeutic decision making. Cancer 2000;88: 2429-2437.

8 Westerterp M, Koppert LB, Buskens CJ, et al. Outcome of surgical treatment for early adenocarcinoma of the esophagus or gastro-esophageal junction. Virchows Arch 2005;446:497-504.

9 Holscher AH, Bollschweiler E, Schneider PM, et al. Early adenocarcinoma in Barrett's oesophagus. Br J Surg 1997;84:1470-1473.

10 Bollschweiler E, Baldus SE, Schroder W, et al. High rate of lymph-node metastasis in submucosal esophageal squamous-cell carcinomas and adenocarcinomas. Endoscopy 2006;3:149-156.

11 Buskens CJ, Westerterp M, Lagarde SM, et al. Prediction of appropriateness of local endoscopic treatment for high-grade dysplasia and early adenocarcinoma by EUS and histopathologic features. Gastrointest Endosc 2004;60:703-710.

12 Cen P, Hofstetter WL, Correa AM, et al. Lymphovascular invasion as a tool to further subclassify T1b esophageal adenocarcinoma. Cancer 2008;112:1020-1027.

13 Nigro JJ, Hagen JA, DeMeester TR, et al. Prevalence and location of nodal metastases in distal esophageal adenocarcinoma confined to the wall: implications for therapy. J Thorac Cardiovasc Surg 1999;117:16-23.

14 Ruol A, Merigliano S, Baldan N, et al. Prevalence, management and outcome of early adenocarcinoma (pT1) of the esophago-gastric junction. Comparison between early cancer in Barrett's esophagus (type I) and early cancer of the cardia (type II). Dis Esophagus 1997;10:190-195.

15 Estrella JS, Hofstetter WL, Correa AM, et al. Duplicated muscularis mucosae invasion has similar risk of lymph node metastasis and recurrence-free survival as intramucosal esophageal adenocarcinoma. Am J Surg Pathol 2011;35:1045-1053. 
16 Pennathur A, Farkas A, Krasinskas AM, et al. Esophagectomy for T1 esophageal cancer: outcomes in 100 patients and implications for endoscopic therapy. Ann Thorac Surg 2009;87:1048-1054.

17 Raja S, Rice TW, Goldblum JR, et al. Esophageal submucosa: the watershed for esophageal cancer. J Thorac Cardiovasc Surg 2011;142:1403-1411, e1.

18 Ancona E, Rampado S, Cassaro M, et al. Prediction of lymph node status in superficial esophageal carcinoma. Ann Surg Oncol 2008;15:3278-3288.

19 Badreddine RJ, Prasad GA, Lewis JT, et al. Depth of submucosal invasion does not predict lymph node metastasis and survival of patients with esophageal carcinoma. Clin Gastroenterol Hepatol 2010;8: 248-253.

20 Gabbert HE, Meier S, Gerharz CD, et al. Tumor-cell dissociation at the invasion front: a new prognostic parameter in gastric cancer patients. Int J Cancer 1992;50:202-207.

21 Prall F. Tumour budding in colorectal carcinoma. Histopathology 2007;50:151-162.

22 Ohike N, Coban I, Kim GE, et al. Tumor budding as a strong prognostic indicator in invasive ampullary adenocarcinomas. Am J Surg Pathol 2010;34:1417-1424.

23 Koike M, Kodera Y, Itoh Y, et al. Multivariate analysis of the pathologic features of esophageal squamous cell cancer: tumor budding is a significant independent prognostic factor. Ann Surg Oncol 2008;15:1977-1982.

24 Brown M, Sillah K, Griffiths EA, et al. Tumour budding and a low host inflammatory response are associated with a poor prognosis in oesophageal and gastro-oesophageal junction cancers. Histopathology 2010;56:893-899.

25 Ueno H, Mochizuki H, Hashiguchi Y, et al. Risk factors for an adverse outcome in early invasive colorectal carcinoma. Gastroenterology 2004;127:385-394.

26 Kalluri R, Weinberg RA. The basics of epithelialmesenchymal transition. J Clin Invest 2009;119: 1420-1428.

27 Zlobec I, Lugli A. Epithelial mesenchymal transition and tumor budding in aggressive colorectal cancer: tumor budding as oncotarget. Oncotarget 2010;1:651-661.

28 Nowak JA, Agoston A, Zheng Y, et al. Tumor budding is a predictor of nodal metastasis and tumor recurrence in T1 esophageal adenocarcinoma. Mod Pathol 2013;26:169A.

29 Boseman FT, Carniero F, Hruban RH, Thiese ND (eds). WHO Classification of Tumors of the Digestive System, 4th edn. IARC: Lyon, 2010.

30 Luketich JD, Pennathur A, Awais O, et al. Outcomes after minimally invasive esophagectomy: review of over 1000 patients. Ann Surg 2012;256:95-103.

31 Guzinska-Ustymowicz K. The role of tumour budding at the front of invasion and recurrence of rectal carcinoma. Anticancer Res 2005;25:1269-1272.

32 Homma Y, Hamano T, Otsuki Y, et al. Severe tumor budding is a risk factor for lateral lymph node metastasis in early rectal cancers. J Surg Oncol 2010;102:230-234.

33 Ishikawa $\mathrm{Y}$, Akishima-Fukasawa $\mathrm{Y}$, Ito $\mathrm{K}$, et al. Histopathologic determinants of regional lymph node metastasis in early colorectal cancer. Cancer 2008;112: 924-933.

34 Kazama S, Watanabe T, Ajioka Y, et al. Tumour budding at the deepest invasive margin correlates with lymph node metastasis in submucosal colorectal cancer detected by anticytokeratin antibody CAM5.2. Br J Cancer 2006;94:293-298.
35 Losi L, Ponti G, Gregorio CD, et al. Prognostic significance of histological features and biological parameters in stage I (pT1 and pT2) colorectal adenocarcinoma. Pathol Res Pract 2006;202:663-670.

36 Sohn DK, Chang HJ, Park JW, et al. Histopathological risk factors for lymph node metastasis in submucosal invasive colorectal carcinoma of pedunculated or semipedunculated type. J Clin Pathol 2007;60: 912-915.

37 Wang LM, Kevans D, Mulcahy H, et al. Tumor budding is a strong and reproducible prognostic marker in T3N0 colorectal cancer. Am J Surg Pathol 2009;33: 134-141.

38 Yamauchi H, Togashi K, Kawamura YJ, et al. Pathological predictors for lymph node metastasis in $\mathrm{T} 1$ colorectal cancer. Surg Today 2008;38:905-910.

39 Yasuda K, Inomata M, Shiromizu A, et al. Risk factors for occult lymph node metastasis of colorectal cancer invading the submucosa and indications for endoscopic mucosal resection. Dis Colon Rectum 2007;50: 1370-1376.

40 Nakamura T, Mitomi H, Kanazawa H, et al. Tumor budding as an index to identify high-risk patients with stage II colon cancer. Dis Colon Rectum 2008;51: $568-572$.

41 Okuyama T, Nakamura T, Yamaguchi M. Budding is useful to select high-risk patients in stage II welldifferentiated or moderately differentiated colon adenocarcinoma. Dis Colon Rectum 2003;46:1400-1406.

42 Okuyama T, Oya M, Ishikawa H. Budding as a useful prognostic marker in pT3 well- or moderately-differentiated rectal adenocarcinoma. J Surg Oncol 2003;83: 42-47.

43 Tanaka M, Hashiguchi $\mathrm{Y}$, Ueno $\mathrm{H}$, et al. Tumor budding at the invasive margin can predict patients at high risk of recurrence after curative surgery for stage II, T3 colon cancer. Dis Colon Rectum 2003;46:1054-1059.

44 Miyata H, Yoshioka A, Yamasaki M, et al. Tumor budding in tumor invasive front predicts prognosis and survival of patients with esophageal squamous cell carcinomas receiving neoadjuvant chemotherapy. Cancer 2009;115:3324-3334.

45 Karamitopoulou E, Zlobec I, Kolzer V, et al. Proposal for a 10-high-power-fields scoring method for the assessment of tumor budding in colorectal cancer. Mod Pathol 2013;26:295-301.

46 Masaki T, Matsuoka H, Sugiyama M, et al. Actual number of tumor budding as a new tool for the individualization of treatment of $\mathrm{T} 1$ colorectal carcinomas. J Gastroenterol Hepatol 2006;21: 1115-1121.

47 Masaki T, Matsuoka H, Sugiyama M, et al. Tumor budding and evidence-based treatment of T2 rectal carcinomas. J Surg Oncol 2005;92:59-63.

48 Morodomi T, Isomoto $\mathrm{H}$, Shirouzu K, et al. An index for estimating the probability of lymph node metastasis in rectal cancers. Lymph node metastasis and the histopathology of actively invasive regions of cancer. Cancer 1989;63:539-543.

49 Mitrovic B, Schaeffer DF, Riddell RH, et al. Tumor budding in colorectal carcinoma: time to take notice. Mod Pathol 2012;25:1315-1325.

50 Altorki NK, Lee PC, Liss Y, et al. Multifocal neoplasia and nodal metastases in T1 esophageal carcinoma: implications for endoscopic treatment. Ann Surg 2008;247:434-439. 
51 Lee L, Ronellenfitsch U, Hofstetter WL, et al. Predicting lymph node metastases in early esophageal adenocarcinoma using a simple scoring system. J Am Coll Surg 2013;217:191-199.

52 Manner H, May A, Pech O, et al. Early Barrett's carcinoma with 'low-risk' submucosal invasion: longterm results of endoscopic resection with a curative intent. Am J Gastroenterol 2008;103:2589-2597.

53 Sepesi B, Watson TJ, Zhou D, et al. Are endoscopic therapies appropriate for superficial submucosal esophageal adenocarcinoma? An analysis of esophagectomy specimens. J Am Coll Surg 2010; 210:418-427.

54 Manner H, Pech O, Heldmann Y, et al. Efficacy, safety, and long-term results of endoscopic treatment for early stage adenocarcinoma of the esophagus with low-risk sm1 invasion. Clin Gastroenterol Hepatol 2013;11: 630-635.

55 Rogers AC, Gibbons D, Hanly AM, et al. Prognostic significance of tumor budding in rectal cancer biopsies before neoadjuvant therapy. Mod Pathol 2014;27: 156-162. 